\title{
Real time observation of gold nanoparticle aggregation dynamics on a 2D membrane
}

Xuezeng Tian ${ }^{1,2}$, Haimei Zheng ${ }^{3}$, Paul Thomas Matsudaira ${ }^{1,2}$ and Utkur Mirsaidov ${ }^{2}$

1. Mechanobiology Institute, National University of Singapore, Singapore, 117411

2. Center for Bioimaging Sciences, Department of Biological Sciences, National University of Singapore, Science Drive 4, Singapore 117543

3. Materials Sciences Division, Lawrence Berkeley National Laboratory, Berkeley, CA 94720

The dynamics of particles confined to the solid-liquid interface is the key to understand the mechanism behind many physical, chemical and biological systems, such as self-assembly, electro-catalysis, and a vast variety of macromolecules on lipid membranes. Recent studies have revealed that the classic Brownian diffusion model and DLVO model have both failed to predict the behaviors regarding the nanoscale interfacial systems, where the surface effects will dominate and regulate the particle motion [1]. It's reported by several authors that nanoparticles on a surface will be damped $10^{-3}-10^{-9}$ times slower than Brownian self-diffusion [2-4]. But how the surface takes effect in particle dynamics is still not fully understood. Thus, a detailed analysis of particle motion could give us more information about the role of surface in regulating particle motion.

We have dedicatedly put high density of gold nanoparticles with typical size $12 \mathrm{~nm}$ on a $\mathrm{SiN}_{\mathrm{x}}$ membrane. The $\mathrm{SiN}_{\mathrm{x}}$ membrane was further assembled into a liquid cell TEM holder for the study. The space between the $\mathrm{SiN}_{\mathrm{x}}$ membranes is filled with distilled water (as Fig. 1a shows). The gold nanoparticles stick to the membrane strongly at the beginning due to van der Waals interaction. When the electron beam is switched on, particles under illumination will start to move vigorously and will aggregate into larger clusters if they meet other particles. It was noted that all the particles move along the membrane and never leave the surface. Such a system resembles a platform to that of 2D aggregation system. We have adopted the classic aggregation theory in analysing the particle aggregation behaviors. We find the aggregation curve on the membrane deviate from the classic diffusion or reaction limited aggregation models, as shown in Fig. 1b and 1c.

Detailed analysis shows the particles movement disobey the Stokes-Einstein law. In our experiments, the translational motion and rotational motion of the clusters are scaled separately with the size growing. We analysed the dynamics of individual clusters, ranging from single particles to very large clusters. Fig. 2 shows a result for early aggregation stage. According to our observation, the small single particles tend to traverse fast along the surface and aggregate into larger clusters, corresponding to the fast aggregation region. When two or more single particles merge into clusters, the clusters exhibit both translational and rotational motion. But the diffusivity of those clusters decrease much faster than that of Stokes-Einstein equation, resulting the bending of the aggregation kinetics from the diffusion limited aggregation model. When the clusters grow larger, they could barely move on the surface, and the aggregation kinetics is further damped. As stated above, the aggregation kinetics curve can be dissected into several regions according to the different diffusing modes of clusters on the surface, as shown in Fig. 3. Different motion modes dominate along with the aggregation process.

Further analysis shows the pinning effect of the surface plays a major role in the dampened motion of clusters. From the dynamic images, we can tell that there are lots of pinning points, as the one shown in the middle of Fig. 2. When a particle or cluster is pinned to the surface, it requires more energy to jump 
out of the pinning point to diffuse. The nature of the pinning points is thought to be strong van der Waals force between particles and $\mathrm{SiN}_{\mathrm{x}}$ membrane. Using Boltzmann equation, we have calculated the energy barrier of the pinning points to be $18 \mathrm{k}_{\mathrm{B}} \mathrm{T}$, where $\mathrm{k}_{\mathrm{B}}$ is Boltzmann constant and $\mathrm{T}$ is temperature.

This work could provide information on the nanoscale dynamics and improve our understanding on solid-liquid interfacial physics.

\section{References:}

[1] F. M. Ross, Science 350 (2015), 6267.

[2] J. Lu et al, Nano Lett. 14 (2014), 2111.

[3] A. Verch et al, Langmuir 31 (2015), 6956.

[4] T. J. Woehl et al, J. Phys. Chem. C 119 (2015), 21261.
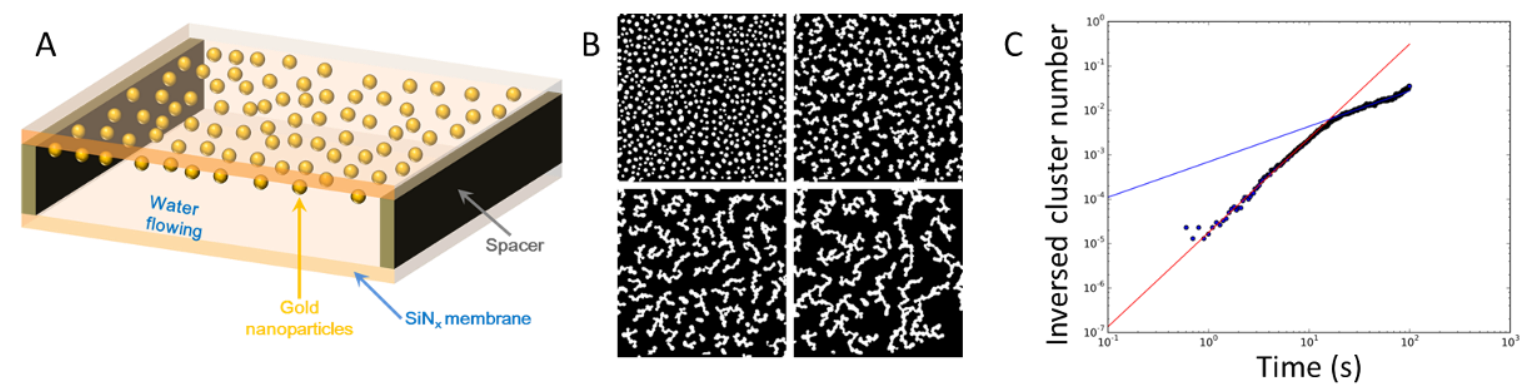

Figure 1. Experimental setup and particle aggregation results. (A) The liquid cell setup. We utilized a hummingbird flow holder to carry out all the experiments. (B) Segmented images showing the aggregation steps. (C) Particle aggregation kinetics curve. Slope for red line (fitting of early stage) is 2.12, while for blue line (fitting of late stage) is 0.80 .

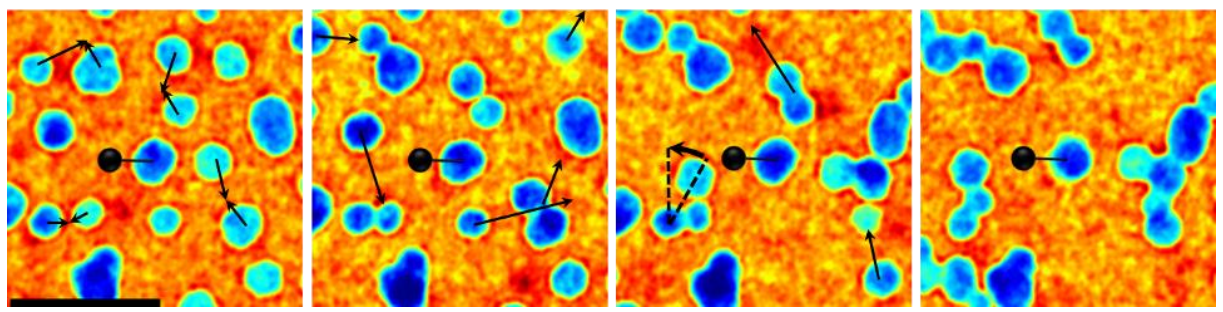

Figure 2. Analysis of individual nanoparticles motion. Arrows indicate the motion of particles/clusters, and dashed lines indicate the rotational angle. Pins show the particle that are pinned to the surface and hardly moves.

Figure 3. Schematic of aggregation kinetics of nanoparticles on a solid-liquid interface. The dashed blue line indicates the aggregation curve of diffusion limited aggregation model, while the solid red line indicates the aggregation curve of particles on a solid-liquid interface. Different motion modes dominate along with the aggregation process.

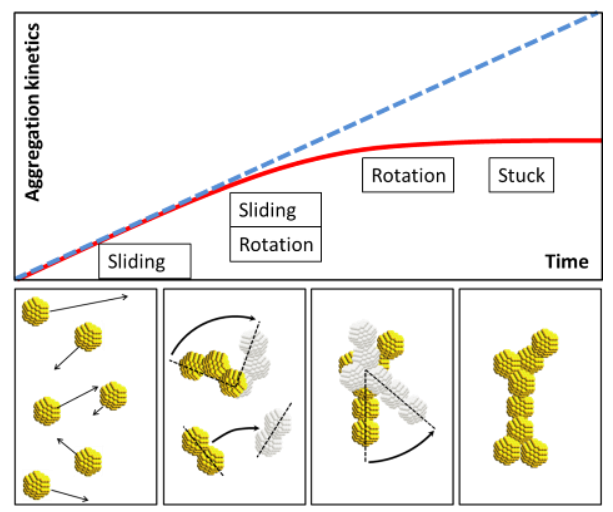

